\section{PHASE-CONTRAST METHODS IN MICROSCOPY}

\author{
By Prof. L. C. MARTIN \\ Imperial College of Science and Technology
}

\begin{abstract}
IF we place, on the stage of a microscope, a slide 1 coated with a thin but opaque metal film pierced by small apertures of distinctive shapes and sizes, there is no doubt as to how its image should appear. We expect to see a dark field surrounding bright areas geometrically similar to the apertures, though on a larger scale. Suppose, however, that the object is a very thin section of some biological structure, for example, a nerve with many component fibres. The various parts of the cellular strueture have little difference in their relative transmission for ordinary light, and only differ optically in their refractive indices. What should the image look like ?
\end{abstract}

It is known from experience with the first type of object that the exact geometrical relations between object and image are most closely preserved when the numerical aperture of the illumination is adequate to fill the aperture of a perfectly corrected and truly focused objective; but if we apply such conditions to the 'section', the image will probably give little indication of any object-structure. The microscope is then telling us that there are no considerable differences of opacity in this object.

It is possible to overcome the difficulty by resorting to methods of 'staining', or using, say, ultra-violet radiation of a wave-length for which the structures may show marked differences of transmission; but both these methods are attended by certain difficulties and disadvantages which need not be detailed here. In spite of their great technical value, they are not always suitable or possible ; thus any method which uses ordinary light, and more or less ordinary microscope equipment, offers much attraction.

Familiar experience shows that if the aperture of the illumination is drastically restricted when viewing such a section, the image will show a pattern in which the distribution of light intensity has at least some geometrical relation with the structure of the object in terms of refractive index, but it is not generally clear how the image is to be interpreted in detail, apart from its broader features. Alternatively, darkground illumination may be employed, in which a pattern is obtained, again with some broadly similar geometrical features, but with marked differences in the expression of detail and with highly accentuated contrasts. In both images any relatively large areas of uniform thickness and refractive index will be rendered uniformly light and dark respectively in the cases of bright and dark fields; the brightness of the image changes only in regions where there are marked variations of optical path in the object.

Phase-contrast offers a means, within certain limits, of relating the brightness to the magnitude of the optical path and not merely to its changes.

Let us confine attention for the time being to a theoretical case in which the illuminating beam is 'parallel', that is, the numerical aperture is zero. Fig. $1 a$ is intended to suggest how the direct light and the diffracted beams meet in the image plane. The diffracted beams are suggested by the broken lines. In such a case it was shown by Lummer that, if the objective aperture were large enough to take in all the light transmitted and diffracted by the object,

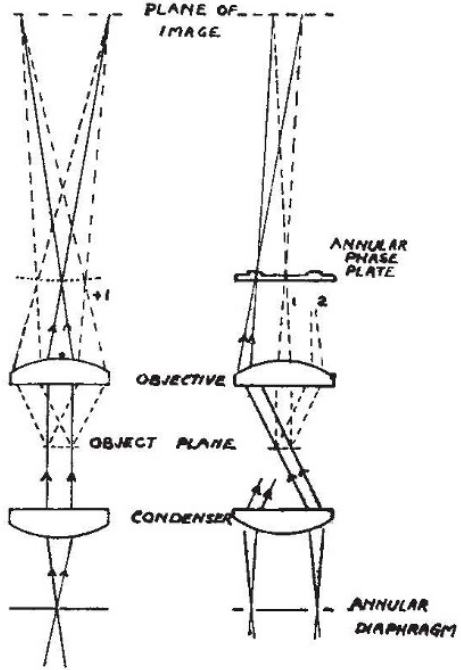

(a)

(b)

the image would represent the object both in intensity and relative phase ; that is, we should see differences of brightness corresponding to the differences of transmission in the object; and moreover, if some parts of the object cause a greater optical path than others, the light in the corresponding parts of the image would exhibit a proportionate retardation of phase. However, this is not immediately helpful as regards visual distinetion, since the eye cannot pick out such phase differences; it can only appreciate differences of brightness in the field.

One of the most interesting contributions to the theory of the interference of light which has been made in recent times is the suggestion of $F$. Zernike that we can render these phase differences visible by adding (or 'subtracting') a uniform background of coherent light. Where the phase of the background agrees with that of the field the brightness will be enhanced; where the phases are opposite there will be a loss of light. We can look on the light leaving the object plane as 'the unaffected wave plus certain diffraction waves produced by the structures'. While the diffracted light is widely spread, the direct light will be brought to something approaching a physical focus in the upper principal focus of the objective before it spreads out to fill the image field and form (together with the 'diffracted' waves) this invisible image expressed in terms of phase variation; but suppose we place in the focus of the direct beam a thin plate of phase-retarding material (a 'phase strip') so small in area that we can neglect without much error its effect on the diffracted waves. Once more we can apply the above idea; the wave leaving the phase strip can be regarded as the incident wave plus a wave due to the strip. We thus add (in effect) a uniform background of coherent light to the original image, and the differences of phase of the image (corresponding to difference of path in the object) become visible as differences of brightness.

The above ideas may be symbolized in the vector diagram, Fig. 2, where $O A$ represents the amplitude and phase of the incident wave; it passes through a certain element of the object and is retarded in phase. The result is now represented by $O P$, which we can look upon as the incident wave $O A$ plus the diffracted wave $O Q$. Again, the incident wave $O A$ passes through the phase strip, where it is retarded by $90^{\circ}$, 


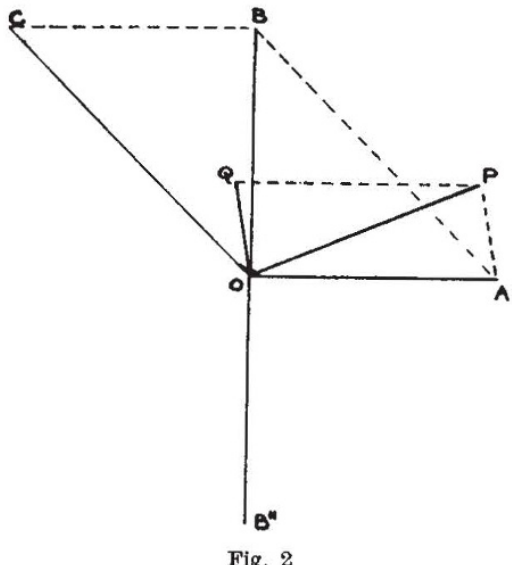

thus becoming $O B$ (note that $O Q$ is regarded as unaffected). Now $O B$ can be represented by $O A$ plus $O C$. Thus the final effect can be looked on as due to: $(a)$ the incident wave (unaffected) $(O A)$; (b) the 'wave' generated by the element $(O Q)$; (c) the 'wave' generated by the phase strip $(O C)$. We can look on the 'wave' $O C$ as adding the 'uniform background of coherent light'. In effect, of course, the resultant is the sum of $O Q$ plus $O B$. This summation is effected in Fig. $3 a$, where the resultant amplitude is represented by $O R$. Suppose now that various parts of the object exhibit regions of small phasechanges for which the 'diffracted' waves in the above sense are $O Q_{1}, O Q_{2}, O Q_{3}$, etc. (Fig. $3 b$ ). Then when the original wave has been subject to the $90^{\circ}$ phase change the resultants will be $O R_{1}, O R_{2}, O R_{3}$, and the corresponding intensities in the image will correspond to the squares of these amplitudes. We shall now have a differing intensity corresponding to the differing phase, and this is an important gain. The relative differences can be enhanced if the amplitude of the direct beam is cut down at the same time that its phase is changed (this can easily be effected by suitable absorbing material, see below). Then its amplitude can be represented by the shortened line $O B^{1}$ (Fig. 3c), and the relative amplitudes of the final resultants by $O R_{1}^{1}, O R_{s}^{1}$, etc., which have a larger relative variation.

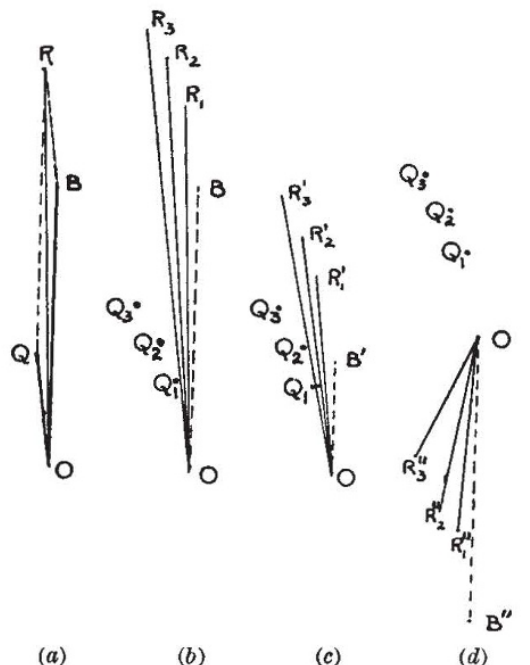

Fig. 3
Again, it is possible so to arrange matters that the phase of the direct light is accelerated with regard to the diffracted waves; then the vector $O A$ (Fig. 2) is shifted to $O B^{11}$, and it will be clear that the resultants $O R^{11}, O R_{2}^{11}$, etc. (Fig. $3 d$ ), have reversed their relative order of magnitude. Hence the contrast is reversed, and the picture will be a negative of the previous one (negative phase-contrast).

The above considerations should help to make clearer the answer to the question which is commonly put regarding phase-contrast. It is this : We are told that in order to establish the best resolution in the image we should employ the widest possible cone of illumination; on the other hand, phase-contrast methods demand a drastic restriction of the numerical aperture of the illumination. Shall we not therefore lose resolution and therewith the truthfulness of the image ?

In answer, it is admitted at once that if the object is one consisting mainly of opacity variation, then the best image is obtained by using the widest possible cone of illumination; if good contrast cannot be obtained with such illumination (as compared with the contrast of the object) then the spherical correction of the objective is suspect, or much stray light may be present. On the other hand, one can only speak of resolving power in relation to a 'phasevariation' object when some means exist of making the structure visible to the eye, with some ascertainable degree of geometrical verisimilitude. If we use a narrow aperture and very oblique illumination, we can, it is true, often obtain evidence of reasonably large phase-variation in fine-structured detail as readily as if a similar opacity structure were present, but if the phase-variations are small the possible brightness differences will be very small also. Using the Abbe principle, it can be said that generally if the angle of a parallel illuminating beam is reduced from the greatest possible obliquity to a direction parallel to the axis, the minimum resolvable interval for a regular structure is increased by a factor of approximately 2; but this depends entirely on the presence of a phase-variation in the object of adequate amount; if the phase-variations are very small, there may not be sufficient contrast in the image to obtain visual resolution of the structure in either case. The discussion of the resolving limit for phase-variation structure has thus no meaning apart from the specification of the phase-angles concerned; for these determine the possible visual contrast.

According to Zernike, phase-contrast has no special use where large phase-variations, say $180^{\circ}$, are concerned; it will, on the other hand, easily render visible phase-differences of very small angle, say $5^{\circ}-10^{\circ}$; moreover, the phase-step is seen as a step of brightness between two uniform corresponding parts of the field, not merely as a halo or dark fringe.

It need not, therefore, be feared that the phasecontrast method must always result in a 'loss of resolution' ; unless, indeed, the object is one of mixed opacity and phase-variation, which is a case requiring special study.

In practice, the simplest arrangement for producing phase-contrast would require a central circular aperture stop in the lower focal plane of the condenser ; this would produce a roughly parallel beam through the object. The phase-plate then occupies a position, in the upper focal plane of the objective, conjugate to the aperture stop. In order to increase the light available for the image, the stop can be elongated to a broad slit; but directional differences then become 


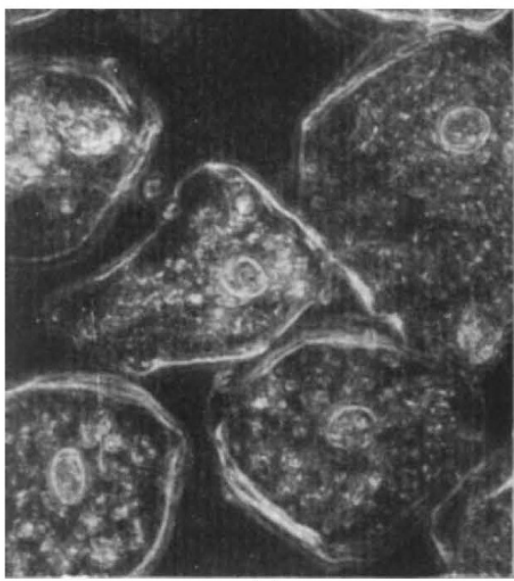

(a)

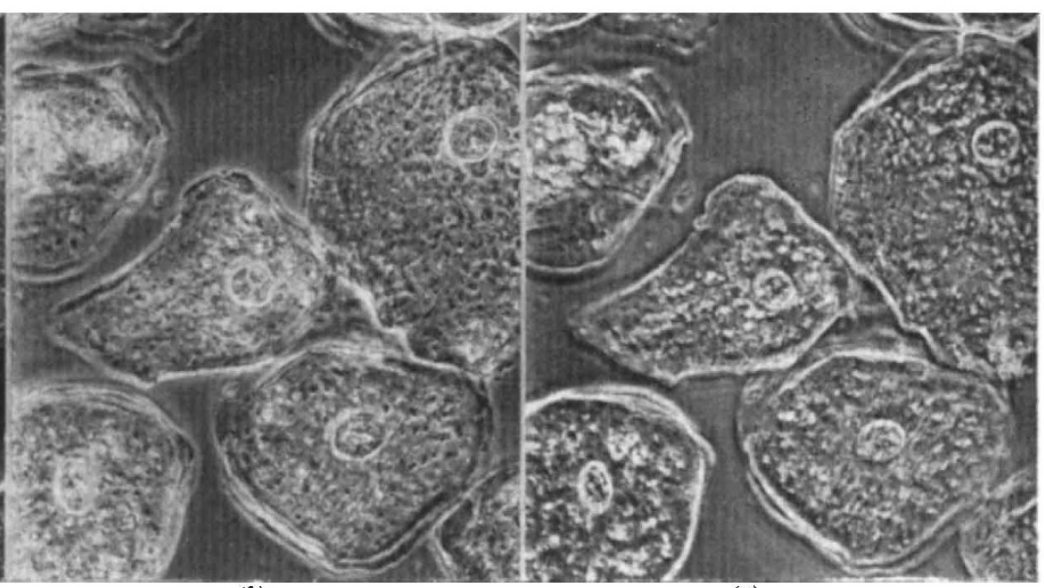

(b) $(c)$

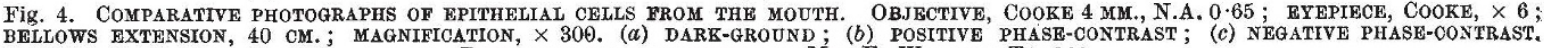
REPRODUCED BX COURTESY OF MR. E. WILFRED TAYLOR

manifest. In practice the most satisfactory form of the condenser stop is thought to be an annular aperture having a mean radius corresponding to about $\frac{1}{2}$ to $\frac{2}{3}$ of the numerical aperture of the objective, and a width about ${ }_{15}$ of this aperture. The phase-plate then has a corresponding phase-changing ring, as suggested in Fig. $1 b$. This arrangement is intended to minimize any loss of resolution.

A retarding ring can be made by vacuum deposition of some suitable material, say calcium fluoride, on the surface of a plane parallel plate; such a ring will usually require protection. Rings can be etched into a similar plate with dilute hydrofluoric acid; and we can thus obtain plates in which the diffracted components suffer greater retardation than the direct light; this is equivalent to an acceleration of the direct light, and would give negative phase-contrast.

If it is desired to diminish the intensity of the zeroorder component, the area of the phase-ring only may be coated with a thin film of metal, say, aluminium, by vacuum distillation.

Alternatively, strips or rings of the desired form can be cut from nitro-cellulose, gelatine, etc., which can be made $3 \mu-6 \mu$ thick ; these may be cemented in a medium having a refractive index differing somewhat from that of the strip. A very precise control of the retardation is thus possible. Gelatine strips can be dyed to give more or less neutral or selective absorption. The upper focal plane of low-power lenses can be furnished with a thin glass plate bearing a suitable ring; but this arrangement will not serve for high powers, where the principal focus may lie within the lens; in such a case the phase-ring may be borne by one of the interior surfaces. It is thus a considerable advantage to have a special set of objectives fitted with such phase-rings. They will not be ideal for use apart from the phase-contrast method, but at any rate if the phase-ring is nonabsorbing the disturbance to the phase-relations in an ordinary image will correspond to the well-known 'Rayleigh Limit', which roughly marks the bounds of discriminable effect. Objectives so fitted can be thus used for ordinary purposes with little risk.

Messrs. Cooke, Troughton, and Simms, Ltd., of York, are producing special sets of objectives, together with the accessory condenser apertures, etc., and the method was recently explained and demonstrated by Mr. E. Wilfred Taylor at a meeting of the Royal Microscopical Society. He showed photographs of biological objects involving the discrimination of extremely small path-differences in flagellæ, etc., which were clearly brought out by phase-contrast. It was elear from such photographs that the images obtained may differ considerably from those obtained by normal methods, and careful attention to the interpretation will be necessary.

Still more recently, Mr. Taylor has made and described a new phase-contrast microseope in which a controlled variable amplitude for the 'direct-light' component is obtained. It is thus possible to adapt the instrumental conditions to suit various types of phase-variation in the object detail. Polarized light is employed, and the necessary relative amplitude variation is secured by the use of a plate, built from concentric annuli of right-handed and left-handed quartz, to carry the phase-changing annulus in the upper focal plane of the objective.

It may be possible perhaps to develop some scheme whereby artificial phase-step objects can be made and included in microscope slides of objects to be studied; the photometric scale for such steps could then be established in terms of the corresponding path differences; this would (for a section of known thick. ness) enable an approximate determination of relative refractive indices to be carried out, which would be a step towards the identification of parts of the structure. Methods of an analogous kind are well known in connexion with doubly refracting materials. The 'artificial objects' could be minute crystallites or photographic step-wedges in gelatine, etc., mounted in balsam of suitable refractive index.

It cannot be said that the practical use of phase. contrast is yet as fully understood as it should be. The theory rests largely on a two-dimensional analysis ; even so, it is only partly developed and the exact effects of the annular phase-contrast rings have not yet been fully explored. During the discussion at the Royal Microscopical Society, Mr. J. R. Smiles, of the National Institute for Medical Research, mentioned difficulties in interpretation of the image due to scattering of light by the object. If the object is an infinitely thin lamina, then the 'scattered' light has the phase characteristics of diffracted light, and consists of the boundary waves, etc., generated in the object plane as well as any light suffering molecular scattering; the effects of this are more or less predictable ; the waves are diffracted at the circular aperture of the objective, and the phase-strip contributes 
its effect also. The general effect is to form fine halo and shadow fringes near the boundaries of phasechange in the object. Zernike has discussed similar effects in his papers, showing how it is that small opaque particles may sometimes appear bright on a dark ground, drawing their light from neighbouring parts of the field.

But the problem grows much more complex when the object is thicker; the sum of the diffracted light may have no easily predictable phase-relation with the incident light, and could thus be spoken of as 'scattered' in that special sense. The halo and fringe effect may therefore be complex, and although the broader regions of uniform phase should still be rendered at the appropriate brightness levels in the image, we cannot interpret the fringes in the same way in every case. The statement above, that the brightness of the image field is related to the retardation of the object, does not apply to the variations of brightness in the border fringes, and the possibility of estimating the retardation does not apply to relatively small objects.

Enough has been said to indicate that the practical technique must be studied in relation to special objects. The simple theory only takes the worker a part of the way, and there is room for a considerable amount of further work both theoretical and practical. However, all the evidence shows that 'phase-contrast' is a most valuable addition to the methods available to the microscopist, and it is being intensively studied at the present time. One of Zernike's own papers (Physica, 9, 686 and 974; 1942) gives the best starting-point.

\section{MARINE BIOLOGY AT PLYMOUTH*}

7 HE Marine Biological Association of the United Kingdom came into being in 1884 as a result of a meeting held under the chairmanship of Prof. T. H. Huxley, the successive outcome of which owed much to the initiative and energy of Sir E. Ray Lankester. The prime purpose of the Association was "the establishment and maintenance of a well-equipped laboratory at a suitable point on the English coast, similar to, if not quite so extensive as, Dr. Dohrn's Zoological Station at Naples". The suitable point finally selected was Plymouth, and there, under the walls of the Citadel built by Charles II and overlooking the waters of Plymouth Sound, the laboratory building, constructed of the same Devonian coral limestone as the Hoe, was opened on June 30, 1888.

Financial support came initially from members of the Association, from universities and other public bodies, notably the Fishmongers' Company, and from H.M. Treasury. All these sources of income have been maintained and increased, but especially the Treasury grant, which became considerable after 1918, when it was put under the control of the Development Commissioners. In 1902 the Association undertook the British share of the scientific investigations of the International Council for the Exploration of the Sea, which involved the establishment of a second laboratory at Lowestoft for work on the trawling grounds and food fishes of the southern North Sea. After 1918 this laboratory was reestablished under the Ministry of Agriculture and Fisheries for work on problems dealing directly with

* Substance of a lecture by Wing-Commander F. S. Russell, F.R.S., delivered at the Royal Society on March 6 . commercial fisheries. The Plymouth Laboratory was to be concerned with the more fundamental problems of marine research, and to this end notable increases were made to the staff, buildings and facilities of the Laboratory in the years between the two World Wars.

The papers published in the Journal of the Association, now in its twenty-sixth volume with two earlier volumes in a smaller format, indicate the changing interests and widening scope of the work carried on in the Laboratory. Earlier volumes are largely concerned with observations on the habits of fishes and shellfish and on the methods and results of commercial fisheries. Aided by enthusiastic visiting workers, the basis was also being laid of the extensive knowledge of the marine life of the Plymouth area now recorded in the "Plymouth Marine Fauna" (second edition, 1931). With the assumption of the work for the International Council opportunities came for initial studies on hydrography, on bottom fauna and on plankton. A notable change comes in the subject-matter of the Journal after 1918, with emphasis on the study of the chemical and physical conditions of the environment, on the life-histories and development of marine invertebrates and on the distribution in space and time of the animal populations. Nevertheless, research on the biology of fishes continued and provided a necessary link with the more economic investigations being carried out at Lowestoft. But recognition of the value of fundamental research had been achieved. To-day we are approaching the stage when all fisheries research will become increasingly fundamental, aimed at understanding the great natural fluctuations in the fish populations and the causes of the habits of the fish themselves.

The Plymouth Laboratory has provided the basis for such far-reaching studies by the concentration of its work around two main themes. The first is how much living matter can the sea produce, what are the variations and causes of variation in productivity, and how do the organisms obtain the materials necessary for life? The second is how do marine animals live, how do they fit their various environments, and what alterations in the conditions in their environment can they appreciate? The sea water contains all the ingredients needed for successful growth and development of living organisms; it is the conditions in the water which therefore determ. ine their distribution and migrations.

The study of the physics and chemistry of the sea has thus become a major preoccupation. The approximate yearly cycle of the more abundant constituents -phosphorus, nitrogen and silicon-is now known, although much remains to be done on the annual turnover and on periodic fluctuations. This in turn involves a study of water movements, especially of upwelling from deep layers. Plant life depends on light and is limited by the depth of its penetration; this is being studied by methods employing photoelectric cells. Meanwhile, pure cultures of diatoms and flagellates are used as experimental material for determining the utilization of combined nitrogen and phosphorus, and the effect of trace elements such as iron and manganese, which may limit plant pro. duction in the sea.

Animal plankton forms the first link of the chain from plant plankton to fish, and researches on its distribution, abundance, growth and habits form a necessary part of the study of the general problem of productivity. The effects of grazing off the plant 